\title{
Racial and Socioeconomic Disparities Are More Pronounced in Inflammatory Breast Cancer Than Other Breast Cancers
}

\author{
Ryan A. Denu, ${ }^{1}$ John M. Hampton, ${ }^{2}$ Adam Currey, ${ }^{3}$ Roger T. Anderson, \\ Rosemary D. Cress, ${ }^{5}$ Steven T. Fleming, ${ }^{6}$ Joseph Lipscomb, ${ }^{7}$ Xiao-Cheng $\mathrm{Wu}^{8}{ }^{8}$ \\ J. Frank Wilson, ${ }^{3}$ and Amy Trentham-Dietz' \\ ${ }^{1}$ Medical Scientist Training Program, University of Wisconsin, 6068 WIMR, 1111 Highland Avenue, Madison, WI 53705, USA \\ ${ }^{2}$ University of Wisconsin Carbone Cancer Center, 610 Walnut Street, Room 307, WARF Building, Madison, WI 53726, USA \\ ${ }^{3}$ Department of Radiation Oncology, Medical College of Wisconsin, 8701 W Watertown Plank Rd, Milwaukee, WI 53226, USA \\ ${ }^{4}$ University of Virginia School of Medicine, West Complex MSB, Room 6203E, 1300 Jefferson Park Ave, Charlottesville, VA 22908, USA \\ ${ }^{5}$ Public Health Institute, Cancer Registry of Greater California, Department of Public Health Sciences, UC Davis School of Medicine, \\ 4610 X St, Davis, CA 95817, USA \\ ${ }^{6}$ College of Public Health, University of Kentucky, 111 Washington Avenue, Lexington, KY 40536, USA \\ ${ }^{7}$ Rollins School of Public Health and Winship Cancer Institute, Emory University, 1518 Clifton Road, Atlanta, GA 30322, USA \\ ${ }^{8}$ LSU Health Sciences Center School of Public Health, 2020 Gravier Street, 3rd Floor, New Orleans, LA 70112, USA \\ ${ }^{9}$ Department of Population Health Sciences, University of Wisconsin Carbone Cancer Center, 610 Walnut Street, Room 307, \\ WARF Building, Madison, WI 53726, USA
}

Correspondence should be addressed to Amy Trentham-Dietz; trentham@wisc.edu

Received 6 April 2017; Accepted 11 July 2017; Published 15 August 2017

Academic Editor: Nicoletta Sacchi

Copyright (C) 2017 Ryan A. Denu et al. This is an open access article distributed under the Creative Commons Attribution License, which permits unrestricted use, distribution, and reproduction in any medium, provided the original work is properly cited.

Inflammatory breast cancer (IBC) is a rare yet aggressive form of breast cancer. We examined differences in patient demographics and outcomes in IBC compared to locally advanced breast cancer (LABC) and all other breast cancer patients from the Breast and Prostate Cancer Data Quality and Patterns of Care Study (POC-BP), containing information from cancer registries in seven states. Out of 7,624 cases of invasive carcinoma, IBC and LABC accounted for $2.2 \%(N=170)$ and $4.9 \%(N=375)$, respectively. IBC patients were more likely to have a higher number $(P=0.03)$ and severity $(P=0.01)$ of comorbidities than other breast cancer patients. Among IBC patients, a higher percentage of patients with metastatic disease versus nonmetastatic disease were black, on Medicaid, and from areas of higher poverty and more urban areas. Black and Hispanic IBC patients had worse overall and breast cancer-specific survival than white patients; moreover, IBC patients with Medicaid, patients from urban areas, and patients from areas of higher poverty and lower education had worse outcomes. These data highlight the effects of disparities in race and socioeconomic status on the incidence of IBC as well as IBC outcomes. Further work is needed to reveal the causes behind these disparities and methods to improve IBC outcomes.

\section{Background}

Inflammatory breast cancer (IBC) is a relatively rare, yet aggressive, subtype of locally advanced breast cancer, with median overall survival less than 4 years [1,2]. IBC incidence ranges from 1 to $6 \%$ of all breast cancers (most recent data suggests 2.5\%) among women in the United States, yet they account for $7 \%$ of all breast cancer deaths [3-9]. Approximately $25 \%$ of IBC patients present with distant disease at diagnosis [4]. IBC was historically a uniformly fatal disease, but the implementation of multifactorial treatments with neoadjuvant chemotherapy, surgery, and radiation has improved survival over the past two decades [10-13]. Response to chemotherapy is one of the strongest predictors of survival [14]. Nevertheless, many patients experience disease recurrence, most frequently to bone, brain, lung, or liver [15]. 
With regard to patient characteristics, IBC patients tend to be younger than other breast cancer patients, with a median age at diagnosis of 57 years for IBC compared to 61.9 for all breast cancers combined $[5,6]$. IBC has been shown to have a higher incidence rate among black women than white women (3.1 per 100,000 women-years for blacks compared to 2.2 for whites) [3]. Further, overall survival has been reported to be significantly worse for blacks than whites [6]. High BMI is associated with an increased risk for IBC compared to nonIBC breast cancer $[16,17]$.

Survival tends to be poorer in IBC patients compared to non-IBC breast cancer patients regardless of histologic subtype $[18,19]$. Data from the National Cancer Institute's Surveillance, Epidemiology, and End Results (SEER) Program indicate that the 5-year survival rate was $34 \%$ for IBC patients from 1988 to 2001 compared to about $87 \%$ for other invasive breast cancers [20]. Although survival for cases of IBC has improved with multimodal therapy [21], recent population statistics still demonstrate much lower median survival for IBC cases (2.9 years) than for cases of locally advanced breast cancer ( 6.4 years) and nonmetastatic breast cancer ( $>10$ years). Therefore, there is great need to identify factors impacting survival in IBC, and the objective of this study was to identify those factors. Using a multistate, population-based sample of patients with IBC, we examined the demographics of these IBC patients compared to patients with locally advanced breast cancer (LABC) because IBC was earlier thought to be a more aggressive form of LABC [10], and $\mathrm{LABC}$ has been used frequently as a comparison group for IBC [22]. Further, we examined differences in IBC patient survival based on patient characteristics.

\section{Materials and Methods}

2.1. Patient Population and Data Sources. The Breast and Prostate Cancer Data Quality and Patterns of Care Study (POC-BP) is the third and most comprehensive POC study from the National Program of Cancer Registries of the Centers for Disease Control and Prevention (CDC). It collected information on breast and prostate cancer patients diagnosed in 2004 in seven states (California, Georgia, Kentucky, Louisiana, Minnesota, North Carolina, and Wisconsin). Cancer registry data were supplemented by reabstracting hospital records and obtaining information about adjuvant treatment and comorbidity from physicians and outpatient facilities and linkages with secondary files such as census data or hospital/physician files. The combined sample size was 9,142 breast cancer patients, from which we extracted patients with IBC $(n=170)$ and compared them to patients with LABC $(n=375)$. Our IBC population was defined as T4d, N0-2, and M0-1. LABC was defined as a diagnosis of stage IIIB or IIIC (this does not include stage III not otherwise specified). As another comparison group, "all else," we included all 9,142 breast cancer patients minus in situ cases and those classified as IBC or LABC $(n=7,079)$.

2.2. Covariates of Interest. Patient race and ethnicity were attained from medical records and categorized into the following groups: non-Hispanic white, non-Hispanic black,
Hispanic, and others. Insurance status at diagnosis was categorized into the following five groups: private, Medicare/other public, Medicaid, none, and unknown. The private insurance group also included cases where the patient had both Medicare and private insurance. "Other public insurance" consisted of patients with TRICARE, other military insurance, Veterans Affairs, or Indian Health Service coverage. The Medicaid group also included women on Medicare with Medicaid eligibility and other government programs. Body mass index (BMI) at the time of diagnosis was categorized into three groups: $<25 \mathrm{~kg} / \mathrm{m}^{2}$ (normal), $25-29 \mathrm{~kg} / \mathrm{m}^{2}$ (overweight), and $\geq 30 \mathrm{~kg} / \mathrm{m}^{2}$ (obese). Area measures were constructed from 2000 US census data linked to the census tract of the patient's residence at the time of diagnosis.

Hospital/institution characteristics were based on the facility in which the patient received breast cancer surgery regardless of the location of other treatments because most referrals for adjuvant therapy are made by a surgeon [23]. If the patient did not receive surgery, then we used the institution where the patient received chemotherapy. If the patient did not receive chemotherapy, then we used the institution where the patient received endocrine therapy.

Patient comorbidities were assessed using the Adult Comorbidity Evaluation-27 (ACE-27) developed by Piccirillo et al. [24]. The ACE-27 was chosen because it reflects a wide range of coexisting conditions and disease severity relevant to cancer therapy choice and outcome.

2.3. Statistical Analysis. Chi-square tests were used to compare IBC to LABC and all other breast cancer patients. Kaplan-Meier survival curves were drawn to compare patients according to different characteristics, with followup beginning at the date of diagnosis through either death or censoring, whichever occurred first. Log-rank tests were calculated to compare survival curves. Cox proportional hazards modeling was used to assess the predictive value of certain factors on the survival of IBC patients. All significance tests were two-sided, and $P$ values less than 0.05 were considered statistically significant. All statistics were weighted by the sampling fractions used by each state registry for the respective sampling stratum to represent the source population. SAS procedures for survey data were used.

\section{Results}

3.1. Demographic and Clinical Characteristics of IBC Patients versus $L A B C$ Patients. We assessed differences in demographics of the patients as well as molecular and clinical characteristics of the disease in IBC compared to LABC and all other breast cancers (Table 1). The mean age of IBC patients was 57.7 years compared to 58.9 for LABC and 59.5 for all others. IBC patients were more likely to be of black race than other breast cancer patients. A greater percentage of IBC patients were obese (BMI > 30) compared to other breast cancer patients. IBC patients were more likely to have comorbidities than other breast cancer patients, and the severity of those comorbidities was also greater for IBC patients compared to other breast cancer patients; no differences in comorbidity number or severity were observed 
TABLE 1: Demographic characteristics of patients with inflammatory breast cancer compared with locally-advanced and all other breast cancer patients, Patterns of Care Study, 2004-2012.

\begin{tabular}{|c|c|c|c|c|c|c|c|c|}
\hline \multirow{2}{*}{ Characteristic } & \multicolumn{2}{|c|}{ IBC } & \multicolumn{2}{|c|}{ LABC (IIIB, C) } & \multirow{2}{*}{$\begin{array}{c}\text { IBC versus LABC } \\
P \text { value }\end{array}$} & \multicolumn{2}{|c|}{ Others } & \multirow{2}{*}{$\begin{array}{c}\text { IBC versus others } \\
P \text { value }\end{array}$} \\
\hline & Number & $\%^{*}$ & Number & $\%^{*}$ & & Number & $\%^{*}$ & \\
\hline Total & 170 & & 375 & & & 7079 & & \\
\hline \multicolumn{9}{|l|}{ Age at diagnosis, years } \\
\hline$<50$ & 50 & 26.4 & 113 & 32.2 & \multirow{4}{*}{0.02} & 2024 & 26.0 & \multirow{4}{*}{0.09} \\
\hline $50-59$ & 49 & 34.1 & 91 & 21.3 & & 1818 & 25.9 & \\
\hline $60-69$ & 36 & 22.6 & 76 & 18.8 & & 1448 & 21.1 & \\
\hline$\geq 70$ & 35 & 16.9 & 95 & 27.6 & & 1789 & 27.0 & \\
\hline \multicolumn{9}{|l|}{ Ancestry/ethnicity } \\
\hline European, non-Hispanic & 81 & 68.4 & 174 & 66.4 & \multirow{4}{*}{0.54} & 4083 & 76.4 & \multirow{4}{*}{0.002} \\
\hline African, non-Hispanic & 68 & 22.4 & 138 & 21.6 & & 1925 & 13.7 & \\
\hline Hispanic & 14 & 7.6 & 38 & 8.2 & & 615 & 6.2 & \\
\hline Other & 7 & 1.6 & 25 & 3.9 & & 456 & 3.8 & \\
\hline \multicolumn{9}{|l|}{ Body mass index $\left(\mathrm{kg} / \mathrm{m}^{2}\right)$} \\
\hline$<25$ & 28 & 16.2 & 88 & 25.5 & \multirow{4}{*}{0.28} & 1743 & 27.0 & \multirow{4}{*}{0.05} \\
\hline $25-29$ & 35 & 24.4 & 77 & 22.1 & & 1626 & 23.5 & \\
\hline$\geq 30$ & 66 & 34.9 & 133 & 34.1 & & 2086 & 27.6 & \\
\hline Unknown & 41 & 24.4 & 77 & 18.4 & & 1624 & 21.9 & \\
\hline \multicolumn{9}{|l|}{ Number of comorbidities } \\
\hline 0 & 58 & 34.8 & 154 & 42.8 & \multirow{4}{*}{0.08} & 3162 & 45.3 & \multirow{4}{*}{0.03} \\
\hline 1 & 57 & 34.3 & 105 & 27.5 & & 2028 & 28.7 & \\
\hline 2 & 35 & 23.2 & 61 & 16.5 & & 1127 & 15.2 & \\
\hline$\geq 3$ & 20 & 7.7 & 55 & 13.2 & & 762 & 10.8 & \\
\hline \multicolumn{9}{|l|}{ Piccirillo Comorbidity Score } \\
\hline None & 55 & 33.0 & 146 & 41.0 & \multirow{5}{*}{0.53} & 2948 & 42.1 & \multirow{5}{*}{0.01} \\
\hline Mild & 68 & 40.6 & 147 & 37.5 & & 2957 & 41.5 & \\
\hline Moderate & 29 & 17.0 & 49 & 14.1 & & 709 & 10.0 & \\
\hline Severe & 16 & 7.9 & 27 & 6.0 & & 287 & 3.8 & \\
\hline Unknown & 2 & 1.6 & 6 & 1.4 & & 178 & 2.6 & \\
\hline \multicolumn{9}{|l|}{ Method of detection } \\
\hline Screening mammography & 8 & 3.8 & 72 & 19.9 & \multirow{5}{*}{$<0.0001$} & 3035 & 44.0 & \multirow{5}{*}{$<0.0001$} \\
\hline Clinical or self-breast exam & 22 & 9.1 & 60 & 16.4 & & 1055 & 13.7 & \\
\hline Self-detection of lump & 58 & 43.3 & 163 & 44.2 & & 2119 & 29.9 & \\
\hline Self-detection, no lump & 74 & 37.1 & 62 & 14.6 & & 399 & 5.2 & \\
\hline Other, unknown & 8 & 6.7 & 18 & 4.9 & & 471 & 7.1 & \\
\hline \multicolumn{9}{|l|}{ Patient health insurance } \\
\hline Private & 77 & 48.4 & 167 & 52.3 & & 4077 & 60.5 & \\
\hline Medicare only/public & 38 & 21.3 & 88 & 24.5 & 0.58 & 1553 & 23.0 & 0.0004 \\
\hline Medicaid & 39 & 20.5 & 84 & 15.4 & 0.00 & 999 & 10.3 & 0.0004 \\
\hline No insurance or unknown & 16 & 9.9 & 36 & 7.8 & & 450 & 6.2 & \\
\hline
\end{tabular}

${ }^{*}$ Percentages weighted based on sampling design. ${ }^{* *} P$ values from chi-square tests.

between IBC and $\mathrm{LABC}$ patients. IBC patients were more likely to be diagnosed by signs or symptoms other than a lump compared to LABC and all other breast cancer patients. In addition, IBC patients were more likely to be from areas with higher poverty compared to other breast cancer patients, but there was no significant difference compared to LABC patients (Table 2). IBC patients were more likely to be treated at large ( $>400$ beds), Commission on Cancer-accredited, teaching hospitals compared to LABC and all other patients.

3.2. Clinical Metastatic versus Nonmetastatic IBC Patient Characteristics. We assessed differences in patient characteristics in IBC patients that presented with clinical metastatic disease $(n=63 ; 37 \%)$ versus nonmetastatic disease $(n=$ 
TABLE 2: Contextual characteristics of patients with inflammatory breast cancer compared with locally advanced and all other breast cancer patients, Patterns of Care Study, 2004-2012.

\begin{tabular}{|c|c|c|c|c|c|c|c|c|}
\hline \multirow{2}{*}{ Characteristic } & \multicolumn{2}{|c|}{ IBC } & \multicolumn{2}{|c|}{ LABC (IIIB, C) } & \multirow{2}{*}{$\begin{array}{l}\text { IBC versus LABC } \\
\quad P \text { value }\end{array}$} & \multicolumn{2}{|c|}{ All else } & \multirow{2}{*}{$\begin{array}{c}\text { IBC versus all else } \\
P \text { value }\end{array}$} \\
\hline & Number & $\%^{*}$ & Number & $\%^{*}$ & & Number & $\%^{*}$ & \\
\hline Total & 170 & & 375 & & & 7079 & & \\
\hline \multicolumn{9}{|l|}{ Poverty (census tract) } \\
\hline$<20 \%$ below poverty & 108 & 73.8 & 257 & 78.1 & \multirow{2}{*}{0.33} & 5280 & 82.3 & \multirow{2}{*}{0.01} \\
\hline$\geq 20 \%$ below poverty & 60 & 25.6 & 117 & 21.4 & & 1779 & 17.4 & \\
\hline \multicolumn{9}{|l|}{ Education (census tract) } \\
\hline$<25 \%$ no high school degree & 83 & 60.2 & 200 & 63.1 & \multirow{2}{*}{0.61} & 4297 & 68.5 & \multirow{2}{*}{0.07} \\
\hline$\geq 25 \%$ no high school degree & 85 & 39.2 & 174 & 36.3 & & 2762 & 31.2 & \\
\hline \multicolumn{9}{|l|}{ Urbanicity } \\
\hline Urban & 94 & 53.8 & 185 & 47.8 & \multirow{4}{*}{0.14} & 3585 & 51.3 & \multirow{4}{*}{0.43} \\
\hline Rural & 23 & 9.2 & 63 & 17.0 & & 1014 & 13.4 & \\
\hline Urban/rural mix & 51 & 36.5 & 126 & 34.7 & & 2461 & 35.0 & \\
\hline Unknown & 2 & 0.5 & 1 & 0.5 & & 19 & 0.3 & \\
\hline \multicolumn{9}{|l|}{ Hospital teaching status } \\
\hline Teaching & 73 & 45.8 & 136 & 37.0 & \multirow{3}{*}{0.06} & 2807 & 39.2 & \multirow{3}{*}{0.10} \\
\hline Other & 66 & 38.2 & 202 & 52.1 & & 3330 & 47.5 & \\
\hline Unknown & 31 & 15.9 & 37 & 10.9 & & 942 & 13.3 & \\
\hline \multicolumn{9}{|l|}{ Hospital size (number of beds) } \\
\hline$<200$ & 32 & 15.1 & 96 & 23.3 & \multirow{5}{*}{0.05} & 1671 & 21.2 & \multirow{5}{*}{0.17} \\
\hline $200-299$ & 20 & 12.4 & 77 & 23.0 & & 1234 & 18.1 & \\
\hline $300-399$ & 21 & 14.7 & 38 & 11.4 & & 842 & 13.2 & \\
\hline$\geq 400$ & 66 & 41.8 & 127 & 31.4 & & 2390 & 34.1 & \\
\hline Unknown & 31 & 15.9 & 37 & 10.9 & & 942 & 13.3 & \\
\hline \multicolumn{9}{|c|}{ Hospital Commission on Cancer Accreditation } \\
\hline No & 59 & 29.3 & 152 & 40.0 & \multirow{3}{*}{0.06} & 2654 & 38.1 & \multirow{3}{*}{0.05} \\
\hline Yes & 87 & 57.8 & 191 & 50.1 & & 3558 & 49.1 & \\
\hline Unknown & 24 & 12.9 & 32 & 9.9 & & 867 & 12.7 & \\
\hline
\end{tabular}

* Percentages weighted based on sampling design. ${ }^{* *} P$ values from chi-square tests.

107; see Supplemental Table 1 in the Supplementary Material available online at https://doi.org/10.1155/2017/7574946). There were no differences in age between metastatic and nonmetastatic IBC patients. Patients presenting with metastatic disease were more likely to be of black race, be insured by Medicaid, and be from areas of higher poverty and more urban areas.

3.3. Factors Predicting Survival of IBC Patients. We compared all-cause and breast cancer-related survival of IBC patients to LABC and all other breast cancer patients. IBC and LABC patients both had significantly worse survival compared to all other breast cancer patients, and IBC patients had significantly worse survival compared to LABC patients (both all-cause and breast cancer-related survival; Supplemental Figure 1). IBC patients of black race and Hispanic ethnicity had worse all-cause and breast cancerrelated survival compared to white IBC patients (Figure 1). We also compared survival based on ER/PR status, finding that IBC patients with ER/PR-positive tumors had better all-cause and breast cancer-specific survival (Supplemental
Figure 2). Lastly, IBC patients who presented with metastatic disease had significantly worse all-cause and breast cancerrelated survival compared to nonmetastatic (Supplemental Figure 3).

Differences in IBC patient survival were also analyzed based on patient characteristics using Cox proportional hazards modeling (Table 3). All Cox models were adjusted for age and metastasis on presentation and weighted based on sampling. Black and Hispanic IBC patients had worse survival compared to white IBC patients; this disparity was not observed in LABC patients. IBC patients with BMI < 25 had 5-year survival rates of $68.5 \%$ compared to $41.6 \%$ for overweight (BMI 25-29) patients and 64.7\% for obese patients $(\mathrm{BMI} \geq 30)$; there were no differences in survival rates of LABC patients based on BMI. Higher number and severity of comorbidities correlated with worse survival rates, both for IBC and for LABC. IBC patients with Medicaid had worse survival than IBC patients with private insurance or Medicare; the same trend was observed in LABC. Furthermore, IBC patients from areas of higher poverty and lower education had worse survival rates, but this was not 

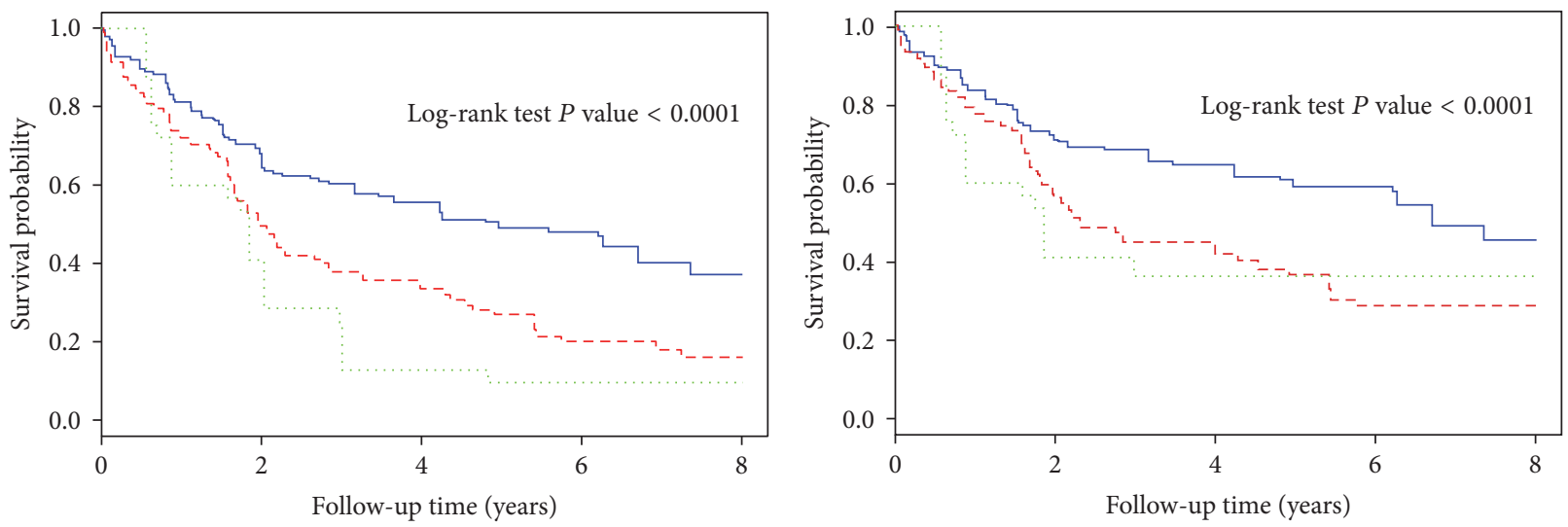

Ancestry/ethnicity
- European
-- African
…. Hispanic

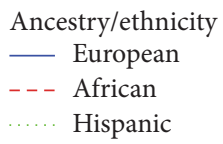

(a)

(b)

FiguRE 1: All-cause overall survival (a) and breast cancer-related survival (b) among inflammatory breast cancer patients based on race and ethnicity, Patterns of Care, 2004-2012.

the case for LABC patients. Patients with metastatic disease on presentation and with ER- and PR- negative tumors had worse survival. Survival was worse in patients with poorly differentiated tumors compared to well or moderately differentiated tumors.

\section{Discussion}

IBC is a rare yet lethal form of LABC. Epidemiological research on IBC has been lacking due to a low incidence of the disease, and most data regarding outcomes of IBC patients are from small single institution series. This Patterns of Care Study with patients from 7 states compared IBC to LABC and all other breast cancers in patient and tumor characteristics and survival. We found that IBC patients had significantly worse survival than $\mathrm{LABC}$ and other breast cancer patients. Our data revealed a 5-year survival rate of $44 \%$ for IBC compared to $58 \%$ for LABC and $84 \%$ for all other cases. This is consistent with other studies [25-27]. Despite the advent of multimodal therapy, including both neoadjuvant and adjuvant chemotherapy, IBC continues to carry a poor prognosis.

The most striking findings in this dataset are the variations in risk and severity of IBC cases based on race and socioeconomic status. A higher percentage of black breast cancer patients have IBC compared to white breast cancer patients. Previous studies have demonstrated similar racial disparities in IBC $[3,4,28-31]$. Furthermore, IBC patients were more likely to be from areas of higher poverty compared to other breast cancer patients. IBC patients presenting with metastatic disease were more likely to be of black race and from poorer, more urban areas. With regard to survival, we found that patients from areas of higher poverty and lower education had worse survival rates. In addition, black and Hispanic IBC patients had worse survival compared to white
IBC patients. Consistent with this, white race has been shown to be an independent predictor of better survival in IBC [32]. It is important for physicians to be cognizant of these disparities so that the causes of such disparities can be better addressed.

The major potential reasons for this disparity in IBC incidence and outcomes based on race and socioeconomic status include (1) decreased access to care; (2) differences in biology due to race or heritage; (3) increased medical mistrust among minorities [33]; and (4) the erythema associated with IBC being less visible in patients with darker skin, potentially leading to a delay in diagnosis [34]. Based on our data and others $[35,36]$, we conclude that the first explanation is most justified; that is, black IBC patients have worse outcomes because they have reduced access to care, not because of biological differences based on race or heritage. Because race/ethnicity and socioeconomic status are so strongly correlated, black patients are less likely to get adequate breast cancer screening, and after diagnosis they generally have more limited access to care, limited treatment options, and difficulty with treatment adherence [37]. Also supporting this claim, Tunisia had a high rate of IBC which has decreased at least in part due to improved socioeconomic status [38].

In our dataset, only $3.8 \%$ of IBC tumors were found by screening mammography. Many patients were diagnosed as a result of symptoms other than a lump. This is consistent with previous reports [15]. There are three hypotheses that could explain this finding. First, it is possible that these women were not getting regular screening mammograms. Secondly, it may be due to the aggressive nature of IBC, suggesting that these tumors developed rapidly between screenings. Lastly, it may be because mammography itself is less effective at detecting these tumors. Some reports have suggested that IBC is difficult to detect using mammography or clinical 
TABLE 3: Hazard ratios for inflammatory breast cancer patient mortality based on patient characteristics, Patterns of Care Study, $2004-2012$.

\begin{tabular}{|c|c|c|c|c|c|c|}
\hline \multirow{3}{*}{ Characteristic } & \multirow{2}{*}{\multicolumn{2}{|c|}{ At risk }} & \multicolumn{4}{|c|}{ Cause of death } \\
\hline & & & \multicolumn{2}{|c|}{ Breast cancer } & \multicolumn{2}{|c|}{ Any cause } \\
\hline & Number & $\%^{*}$ & Number of deaths & $\operatorname{HR}(95 \% \mathrm{CI})^{* *}$ & Number of deaths & $\operatorname{HR}(95 \% \mathrm{CI})^{* *}$ \\
\hline Total & 170 & & 91 & & 121 & \\
\hline \multicolumn{7}{|l|}{ Age at diagnosis, years } \\
\hline$<50$ & 50 & 26.4 & 25 & 1 (ref.) & 30 & 1 (ref.) \\
\hline $50-59$ & 49 & 34.1 & 31 & $2.05(1.03-4.08)$ & 37 & $1.89(1.03-3.47)$ \\
\hline $60-69$ & 36 & 22.6 & 18 & $1.15(0.48-2.74)$ & 24 & $1.28(0.65-2.53)$ \\
\hline$\geq 70$ & 35 & 16.9 & 17 & $1.92(0.81-4.56)$ & 30 & $2.53(1.34-4.77)$ \\
\hline \multicolumn{7}{|l|}{ Ancestry/ethnicity } \\
\hline European, non-Hispanic & 81 & 68.4 & 36 & 1 (ref.) & 48 & 1 (ref.) \\
\hline African, non-Hispanic & 68 & 22.4 & 43 & $1.76(1.05-2.97)$ & 56 & $1.86(1.19-2.91)$ \\
\hline Hispanic & 14 & 7.6 & 8 & $1.14(0.35-3.71)$ & 11 & $1.57(0.72-3.40)$ \\
\hline Other & 7 & 1.6 & 4 & $1.25(0.67-2.33)$ & 6 & $1.66(0.88-3.13)$ \\
\hline \multicolumn{7}{|l|}{ Piccirillo Comorbidity Score } \\
\hline None & 55 & 33.0 & 26 & 1 (ref.) & 35 & 1 (ref.) \\
\hline Mild & 68 & 40.6 & 38 & $0.55(0.27-1.12)$ & 48 & $0.48(0.27-0.87)$ \\
\hline Moderate & 29 & 17.0 & 14 & $1.01(0.43-2.41)$ & 21 & $0.86(0.39-1.86)$ \\
\hline Severe & 16 & 7.9 & 13 & $2.77(1.35-5.69)$ & 16 & $2.64(1.32-5.27)$ \\
\hline Unknown & 2 & 1.6 & 0 & - & 1 & $0.88(0.44-1.75)$ \\
\hline \multicolumn{7}{|l|}{ Metastasis on presentation } \\
\hline Yes & 63 & 31.1 & 49 & $3.20(1.87-5.48)$ & 59 & $2.97(1.86-4.73)$ \\
\hline No & 107 & 68.9 & 42 & 1 (ref.) & 62 & 1 (ref.) \\
\hline \multicolumn{7}{|l|}{ ER/PR status } \\
\hline $\mathrm{ER}+$ and/or PR+ & 87 & 49.1 & 38 & $0.55(0.29-1.03)$ & 56 & $0.54(0.32-0.92)$ \\
\hline ER- and PR- & 70 & 42.9 & 45 & 1 (ref.) & 54 & 1 (ref.) \\
\hline Unknown & 13 & 8.1 & 8 & $2.11(0.76-5.83)$ & 11 & $1.89(0.71-5.05)$ \\
\hline \multicolumn{7}{|l|}{ HER2 status } \\
\hline Positive & 62 & 35.9 & 33 & $0.74(0.40-1.36)$ & 42 & $0.90(0.55-1.49)$ \\
\hline Negative & 83 & 48.2 & 45 & 1 (ref.) & 61 & 1 (ref.) \\
\hline Unknown & 25 & 16.0 & 13 & $0.84(0.19-3.70)$ & 18 & $0.80(0.21-3.07)$ \\
\hline \multicolumn{7}{|l|}{ Grade } \\
\hline Well or moderately differentiated & 31 & 22.4 & 13 & 1 (ref.) & 21 & 1 (ref.) \\
\hline Poorly differentiated & 112 & 66.0 & 64 & $1.84(0.72-4.71)$ & 84 & $1.83(0.91-3.69)$ \\
\hline Undifferentiated & 6 & 1.8 & 2 & $0.46(0.05-3.91)$ & 2 & $0.38(0.05-2.81)$ \\
\hline Unknown & 21 & 9.9 & 12 & $1.12(0.39-3.20)$ & 14 & $0.84(0.36-1.96)$ \\
\hline
\end{tabular}

ER, estrogen receptor; HR, hazard ratio; PR, progesterone receptor; ref., reference group. ${ }^{*}$ Percentages weighted based on sampling design. ${ }^{* *}$ Cox proportional hazards models adjusted for age, metastasis on presentation, ER/PR status, treatment, and sampling weights and strata within each study center.

breast exam because there is often no mass [2]. When there is a mass, it is often masked by diffusely increased breast density. IBC is found in a disproportionate number of women of low socioeconomic status, and breast cancer screening is generally lower in people of lower socioeconomic status [39].

IBC patients tend to have worse comorbidities than other breast cancer patients. One study found that women with endocrine disease, psychiatric disorders, and hematologic disease were more likely to be diagnosed with advanced breast cancer, while those with cardiovascular disease, musculoskeletal disorders, gastrointestinal disease, benign breast disease, and genitourinary disorders were less likely to be diagnosed with advanced breast cancer after controlling for number of physician visits [40]. The risk for death among breast cancer patients depends heavily on the stage of illness at diagnosis, and comorbid illnesses are thought to increase the risk of late stage disease for a number of reasons [40, 41]. Some suggest that early symptoms of breast cancer are sometimes confused with the symptoms of comorbid illnesses or are less significant than other health problems [42]. Women with comorbidities are more likely to access health care and therefore may be more likely to get screened for breast cancer [43]. However, some studies suggest that comorbidities can be a barrier to breast cancer screening [44]; our data tends to support this hypothesis. Lastly, there may be some interaction between certain comorbidities and the development of cancer; for example, diabetes is thought to 
increase risk of cancer via hyperinsulinemia, hyperglycemia, and inflammation [45].

A major strength of this study is its large-scale population-based design, including data from seven states. Other strengths were the inclusion of National Program of Cancer Registries that did not participate in previous POC studies and comprehensive treatment information. Limitations include the small number of IBC cases, as all past IBC research has been hampered by this as well.

\section{Conclusion}

In conclusion, this study highlights potential reasons for disparities in IBC incidence and severity, which represent targetable-albeit not modifiable-factors. We identify disparities in race and socioeconomic status on the risk of IBC as well as IBC outcomes. Further work is needed to reveal the causes behind these disparities and methods to improve IBC outcomes.

\section{Disclosure}

This article was written on behalf of the Patterns of Care Study BP Group. The findings and conclusions in this report are those of the authors and do not necessarily represent the official position of the Centers for Disease Control and Prevention.

\section{Conflicts of Interest}

The authors declare that there are no conflicts of interest regarding the publication of this article.

\section{Acknowledgments}

Ryan A. Denu is supported by the University of Wisconsin Medical Scientist Training Program (T32GM008692) and the University of Wisconsin ICTR TL1 Training Program (supported under NIH Awards UL1TR000427 and TL1TR000429). The data used for this publication were collected by the CDC National Program of Cancer Registries Patterns of Care Study for Female Breast and Prostate Cancers through cooperative agreements with the participating state cancer registries including Grant U01DP000261 to J. Frank Wilson. This study was also supported in part by NIH Grant P30CA014520.

\section{References}

[1] J. A. Low, A. W. Berman, S. M. Steinberg, D. N. Danforth, M. E. Lippman, and S. M. Swain, "Long-term follow-up for locally advanced and inflammatory breast cancer patients treated with multimodality therapy," Journal of Clinical Oncology, vol. 22, no. 20, pp. 4067-4074, 2004.

[2] S. D. Merajver, M. D. Iniesta, and M. S. Sabel, Inflammatory Breast Cancer, Lippincott Williams \& Wilkins, Philadelphia, Pa, USA, 4th edition, 2010.

[3] K. W. Hance, W. F. Anderson, S. S. Devesa, H. A. Young, and P. H. Levine, "Trends in inflammatory breast carcinoma incidence and survival: the surveillance, epidemiology, and end results program at the National Cancer Institute," Journal of the National Cancer Institute, vol. 97, no. 13, pp. 966-975, 2005.

[4] P. A. Wingo, P. M. Jamison, J. L. Young, and P. Gargiullo, "Population-based statistics for women diagnosed with inflammatory breast cancer (United States)," Cancer Causes and Control, vol. 15, no. 3, pp. 321-328, 2004.

[5] W. F. Anderson, C. Schairer, B. E. Chen, K. W. Hance, and P. H. Levine, "Epidemiology of inflammatory breast cancer (IBC)," Breast Disease, vol. 22, pp. 9-23, 2005.

[6] S. Chang, S. L. Parker, T. Pham, A. U. Buzdar, and S. D. Hursting, "Inflammatory breast carcinoma incidence and survival: The Surveillance, Epidemiology, and End Results program of the National Cancer Institute, 1975-1992," Cancer, vol. 82, no. 12, pp. 2366-2372, 1998.

[7] L. H. Stocks and F. M. Patterson, "Inflammatory carcinoma of the breast," Surgery, Gynecology \& Obstetrics, vol. 143, no. 6, pp. 885-889, 1976.

[8] P. H. Levine, S. C. Steinhorn, L. Gloeckler Ries, and J. L. Aron, "Inflammatory breast cancer: The experience of the surveillance, epidemiology, and end results (SEER) program," Journal of the National Cancer Institute, vol. 74, no. 2, pp. 291297, 1985.

[9] I. A. Jaiyesimi, A. U. Buzdar, and G. Hortobagyi, "Inflammatory breast cancer: A review," Journal of Clinical Oncology, vol. 10, no. 6, pp. 1014-1024, 1992.

[10] S. Dawood and M. Cristofanilli, "Inflammatory breast cancer: What progress have we made?” Oncology (Williston Park, N.Y.), vol. 25, no. 3, pp. 264-273, 2011.

[11] N. T. Ueno, A. U. Buzdar, S. E. Singletary et al., "Combinedmodality treatment of inflammatory breast carcinoma: Twenty years of experience at M.D. Anderson Cancer Center," Cancer Chemotherapy and Pharmacology, vol. 40, no. 4, pp. 321-329, 1997.

[12] S. Rehman, C. A. Reddy, and R. D. Tendulkar, "Modern outcomes of inflammatory breast cancer," International Journal of Radiation Oncology Biology Physics, vol. 84, no. 3, pp. 619-624, 2012.

[13] S. Dawood, X. Lei, R. Dent et al., "Survival of women with inflammatory breast cancer: A large population-based study," Annals of Oncology, vol. 25, no. 6, pp. 1143-1151, 2014.

[14] H. J. Hoffman, A. Khan, K. M. Ajmera, L. Zolfaghari, J. R. Schenfeld, and P. H. Levine, "Initial response to chemotherapy, not delay in diagnosis, predicts overall survival in inflammatory breast cancer cases," American Journal of Clinical Oncology: Cancer Clinical Trials, vol. 37, no. 4, pp. 315-321, 2014.

[15] J. M. Matro, T. Li, M. Cristofanilli et al., "Inflammatory breast cancer management in the National Comprehensive Cancer Network: The disease, recurrence pattern, and outcome," Clinical Breast Cancer, vol. 15, no. 1, pp. 1-7, 2015.

[16] S. Chang, A. U. Buzdar, and S. D. Hursting, "Inflammatory breast cancer and body mass index," Journal of Clinical Oncology, vol. 16, no. 12, pp. 3731-3735, 1998.

[17] S. I. Labidi, K. Mrad, A. Mezlini et al., "Inflammatory breast cancer in Tunisia in the era of multimodality therapy," Annals of Oncology, vol. 19, no. 3, pp. 473-480, 2008.

[18] S. Chang, J. R. Alderfer, L. Asmar, and A. U. Buzdar, "Inflammatory breast cancer survival: The role of obesity and menopausal status at diagnosis," Breast Cancer Research and Treatment, vol. 64, no. 2, pp. 157-163, 2000. 
[19] K. Raghav, J. T. French, N. T. Ueno et al., "Inflammatory breast cancer: A distinct clinicopathological entity transcending histological distinction," PLoS ONE, vol. 11, no. 1, Article ID e0145534, 2016.

[20] J. M. Goldfarb and J. E. Pippen, "Inflammatory breast cancer: the experience of Baylor University Medical Center at Dallas," in Proceedings of the (Baylor University Medical Center), vol. 24, pp. 86-88.

[21] P. Tai, E. Yu, R. Shiels et al., "Short- and long-term causespecific survival of patients with inflammatory breast cancer," BMC Cancer, vol. 5, article no. 137, 2005.

[22] S. Chia, S. M. Swain, D. R. Byrd, and D. A. Mankoff, "Locally advanced and inflammatory breast cancer," Journal of Clinical Oncology, vol. 26, no. 5, pp. 786-790, 2008.

[23] L. A. Siminoff, A. Zhang, C. M. Saunders Sturm, and N. Colabianchi, "Referral of breast cancer patients to medical oncologists after initial surgical management," Medical Care, vol. 38, no. 7, pp. 696-704, 2000.

[24] J. F. Piccirillo, C. Creech, R. Zequeira, S. Anderson, and A. S. Johnston, "Inclusion of comorbidity into oncology data registries," Journal of Registry Management, vol. 26, pp. 66-70, 1999.

[25] A. M. Gonzalez-Angulo, B. T. Hennessy, K. Broglio et al., "Trends for inflammatory breast cancer: Is survival improving?" Oncologist, vol. 12, no. 8, pp. 904-912, 2007.

[26] American Joint Committee on Cancer, "Breast," in AJCC Cancer Staging Manual, F. L. Greene, D. L. Page, I. D. Fleming et al., Eds., vol. 6, Springer-Verlag, New York, NY, USA, 2002.

[27] S. Dawood, N. T. Ueno, V. Valero et al., "Differences in survival among women with stage III inflammatory and noninflammatory locally advanced breast cancer appear early," Cancer, vol. 117, no. 9, pp. 1819-1826, 2011.

[28] J. K. Schinkel, S. H. Zahm, I. Jatoi et al., "Racial/ethnic differences in breast cancer survival by inflammatory status and hormonal receptor status: An analysis of the Surveillance, Epidemiology, and End Results data," Cancer Causes and Control, vol. 25, no. 8, pp. 959-968, 2014.

[29] J. A. Schlichting, A. S. Soliman, C. Schairer et al., "Association of inflammatory and noninflammatory breast cancer with socioeconomic characteristics in the surveillance, epidemiology, and end results database, 2000-2007," Cancer Epidemiology Biomarkers and Prevention, vol. 21, no. 1, pp. 155-165, 2012.

[30] J. A. Schlichting, A. S. Soliman, C. Schairer, D. Schottenfeld, and S. D. Merajver, "Inflammatory and non-inflammatory breast cancer survival by socioeconomic position in the Surveillance, Epidemiology, and End Results database, 1990-2008," Breast Cancer Research and Treatment, vol. 134, no. 3, pp. 1257-1268, 2012.

[31] D. Il'yasova, S. Siamakpour-Reihani, I. Akushevich, L. Akushevich, N. Spector, and J. Schildkraut, "What can we learn from the age- and race/ethnicity- specific rates of inflammatory breast carcinoma?" Breast Cancer Research and Treatment, vol. 130, no. 2, pp. 691-697, 2011.

[32] S. Dawood, N. T. Ueno, V. Valero et al., "Identifying factors that impact survival among women with inflammatory breast cancer," Annals of Oncology, vol. 23, no. 4, pp. 870-875, 2012.

[33] N. A. Bickell, J. Weidmann, K. Fei, J. J. Lin, and H. Leventhal, "Underuse of breast cancer adjuvant treatment: Patient knowledge, beliefs, and medical mistrust," Journal of Clinical Oncology, vol. 27, no. 31, pp. 5160-5167, 2009.
[34] F. M. Robertson, M. Bondy, W. Yang et al., "Inflammatory breast cancer the disease, the biology, the treatment," CA Cancer Journal for Clinicians, vol. 60, no. 6, pp. 351-375, 2010.

[35] O. W. Brawley, "Toward a better understanding of race and cancer," Clinical Cancer Research, vol. 16, no. 24, pp. 5920-5922, 2010.

[36] O. W. Brawley, "Health disparities in breast cancer," Obstetrics and Gynecology Clinics of North America, vol. 40, no. 3, pp. 513$523,2013$.

[37] A. Bhargava and X. L. Du, "Racial and socioeconomic disparities in adjuvant chemotherapy for older women with lymph node-positive, operable breast cancer," Cancer, vol. 115, no. 13, pp. 2999-3008, 2009.

[38] H. Boussen, H. Bouzaiene, J. B. Hassouna et al., "Inflammatory breast cancer in Tunisia: Epidemiological and clinical trends," Cancer, vol. 116, no. 11, pp. 2730-2735, 2010.

[39] M. E. Peek and J. H. Han, "Disparities in screening mammography: Current status, interventions, and implications," Journal of General Internal Medicine, vol. 19, no. 2, pp. 184-194, 2004.

[40] S. T. Fleming, H. G. Pursley, B. Newman, D. Pavlov, and K. Chen, "Comorbidity as a predictor of stage of illness for patients with breast cancer," Medical Care, vol. 43, no. 2, pp. 132-140, 2005.

[41] C. J. Newschaffer, T. L. Bush, L. E. Penberthy, M. Bellantoni, K. Helzlsour, and M. Diener-West, "Does comorbid disease interact with cancer? An epidemiologic analysis of mortality in a cohort of elderly breast cancer patients," Journals of Gerontology - Series A Biological Sciences and Medical Sciences, vol. 53, no. 5, pp. M372-M378, 1998.

[42] W. A. Satariano, "Comorbidity and functional status in older women with breast cancer: Implications for screening, treatment, and prognosis," Journals of Gerontology, vol. 47, pp. 24-31, 1992.

[43] P. A. Vaeth, W. A. Satariano, and D. R. Ragland, "Limiting Comorbid Conditions and Breast Cancer Stage at Diagnosis," The Journals of Gerontology Series A: Biological Sciences and Medical Sciences, vol. 55, no. 10, pp. M593-M600, 2000.

[44] C. I. Kiefe, E. Funkhouser, M. N. Fouad, and D. S. May, "Chronic disease as a barrier to breast and cervical cancer screening," Journal of General Internal Medicine, vol. 13, no. 6, pp. 357-365, 1998.

[45] E. Giovannucci, D. M. Harlan, M. C. Archer et al., "Diabetes and cancer: a consensus report," Diabetes Care, vol. 33, no. 7, pp. 1674-1685, 2010. 


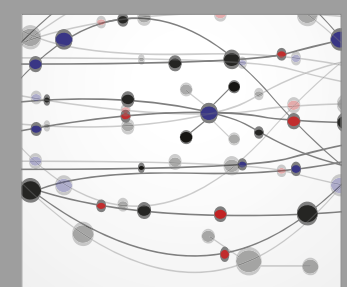

The Scientific World Journal
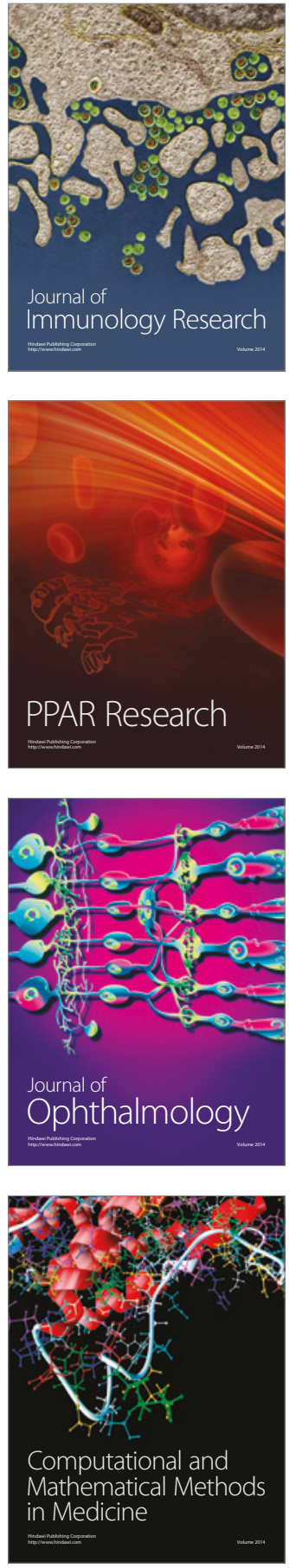

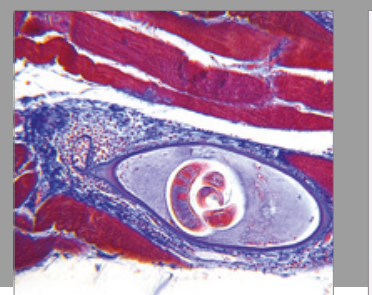

Gastroenterology Research and Practice
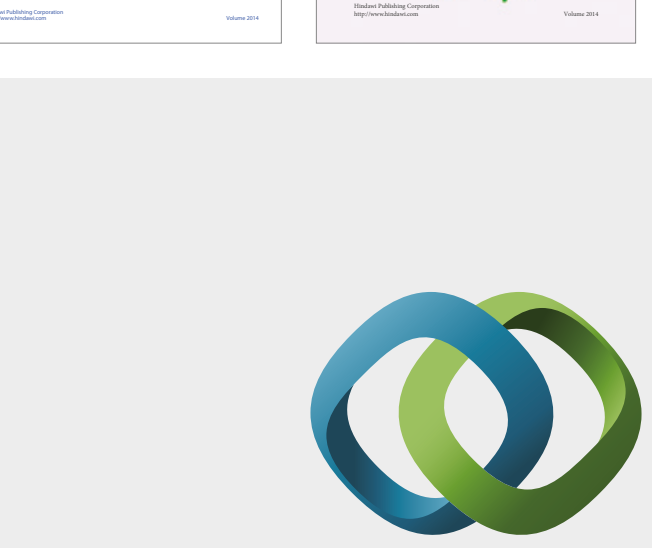

\section{Hindawi}

Submit your manuscripts at

https://www.hindawi.com
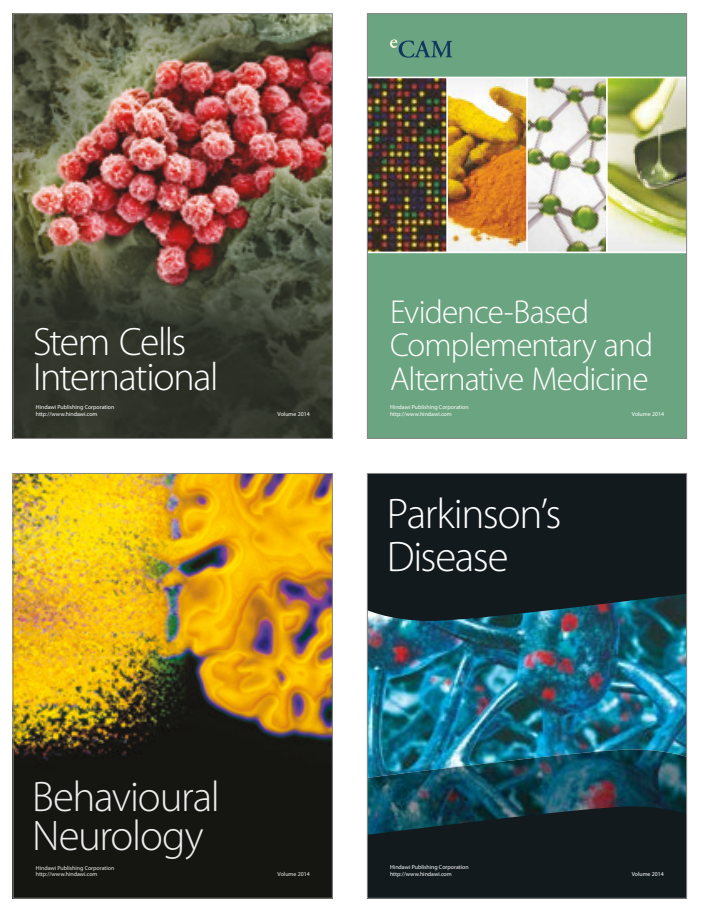
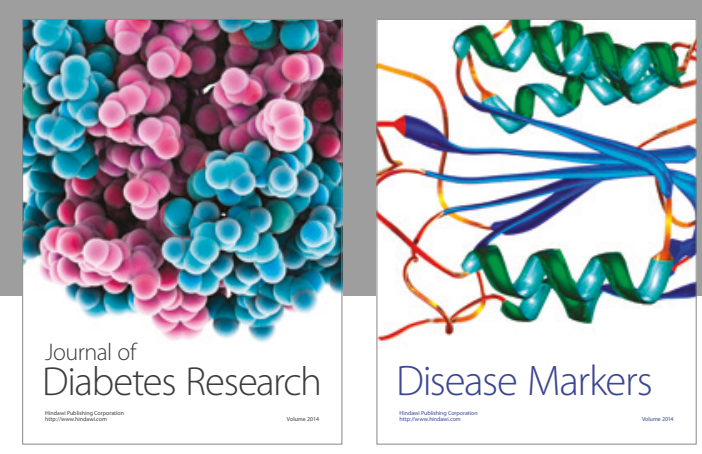

Disease Markers
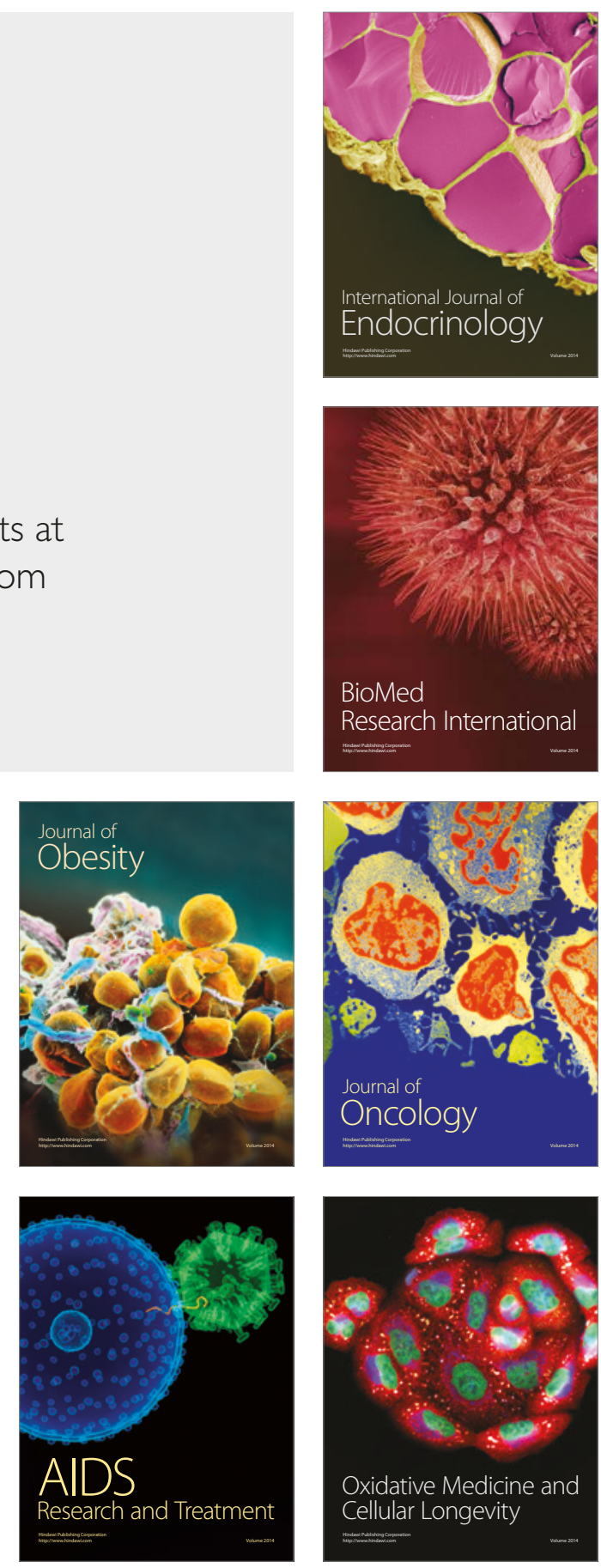